\section{Numerical Simulation of 3D Cavitating Flows: Analysis of Cavitation Head Drop in Turbomachinery}

The numerical simulation of cavitating flows in turbomachinery is studied at the Turbomachinery and Cavitation team of Laboratoire des Ecoulements Géophysiques et Industriels (LEGI), Grenoble, France in collaboration with the French space agency (Centre National d'Etudes Spatiales, CNES), the rocket engine division of Snecma and Numeca International. A barotropic state law is proposed to model the cavitation phenomenon and this model has been integrated in the CFD code FINE/TURBO ${ }^{\text {TM }}$. An analysis methodology allowing the numerical simulation of the head drop induced by the development of cavitation in cold water was proposed and applied in the case of two four-bladed inducers and one centrifugal pump. Global results were compared to available experimental results. Internal flows in turbomachinery were investigated in depth. Numerical simulations enabled the characterization of the mechanisms leading to the head drop and the visualization of the effects of the development of cavitation on internal flows.

\author{
Benoît Pouffary \\ Centre National d'Etudes Spatiales, \\ Evry, 91023 France \\ Regiane Fortes Patella
INPG-LEGI,
Grenoble, 38041 France
}

Jean-Luc Reboud

CNRS-G2ELAB,

University of Grenoble,

Grenoble, 38042 France

Pierre-Alain Lambert
Snecma,
Vernon, 27208 France

\section{Introduction}

Cavitation is an important phenomenon for the design of rocket engine turbopumps and remains a major issue for the space industry. That is why the CNES and the Rocket Engine Division of Snecma have supported research in order to make progress in the understanding and prediction of the mechanisms associated with cavitation for many years. In this frame, one main purpose of the research performed at the Turbomachinery and Cavitation team of Laboratoire des Ecoulements Géophysiques et Industriels (LEGI) consists in developing and validating a 3D Reynolds-averaged Navier-Stokes (RANS) code, enabling the simulation of the cavitating operation of turbopumps. Through collaboration between LEGI and Numeca International, a physical model of cavitation was implemented in the commercial code FINE/TURBO ${ }^{\mathrm{TM}}$ [1-3].

The applied numerical model is very similar to the one proposed by Kunz et al. $[4,5]$ to calculate cavitating flows in centrifugal pumps. The main difference between the present work and those studies is the physical model applied to describe the cavitation phenomenon. In this study, the barotropic cavitation model initially proposed by Delannoy and Kueny [6] is considered and presented in the first section of this paper.

The 3D RANS code, described in Sec. 3, allows the steady and unsteady cavitating flow calculations in the $2 \mathrm{D}$ or $3 \mathrm{D}$ geometries, as presented in Refs. [1-3,7], and could be used as a tool to analyze inducer instabilities [8].

In complement to previous work $[1,7,9]$, the main target of the present study is to propose a qualitative and quantitative analysis of the cavitation head drop in turbomachinery. As a matter of fact, some recent numerical works have been developed to predict cavitation inception, cavity dimensions, and/or the threshold corresponding to pump head drops. For example, Ait-Bouziad et al. $[10,11]$ applied off-the-shelf software to investigate cavitating flows in a three-bladed industrial inducer. The authors present interesting global and local analyses of the cavitating flow field, but they noticed that the absolute value of head drop was not well predicted by the numerical simulations. Mejri et al. [12] applied the same off-the-shelf software to analyze the overall performances, as well as the location and sizes of blade cavities for the three three-bladed inducers identified by different blade angles at the leading edge. A qualitative agreement between the experimental and predicted results was found for two inducers. The authors concluded that the implemented cavitation model overpredicted the cavitating flow, mainly in partial load conditions.

We cite also the important numerical work developed by Hosangadi and Ahuja [13] concerning cavitating flows over a hydrofoil and a cylindrical headform. In Ref. [14], Hosangadi et al. presented a good comparison between the simulated and experimental data concerning breaking down of a helical flat-plate inducer configuration in cold water.

In spite of those relevant works, more studies are needed to improve and validate simulated and physical approaches in order to obtain reliable quantitative predictions of overall performances, especially in the case of inducer geometries. Moreover, detailed cavitating flow analyses are also required for the better understanding of the head drop hydrodynamic mechanism and of energy transfers in centrifugal and axial pumps.

In this context, the developed numerical model has been applied to calculate 3D steady cavitating flows in a centrifugal pump and in two rocket engine turbopump inducers, whose geometries are described in Sec. 4. The influence of steady cavitation behavior on the pump characteristics and on the final head drops has been simulated and compared to experimental results for different operating points. The observations of the pump global performances and associated local flow fields led us to carry out a detailed analysis of the influence of cavitation in the three different geometries (Sec. 5). From these results, the origin of the cavitation head drop is discussed and an analysis of its mechanism is proposed. The study is improved by the investigation of the repartition of the total pressure rise, the blade loads, as well as by the evaluation of cavitation influence on secondary flows.

\section{Barotropic State Law}

The considered numerical approach uses a single fluid model to describe the liquid-vapor mixture, with a mixture density $\rho$ varying in the flow field between the vapor density and the liquid 


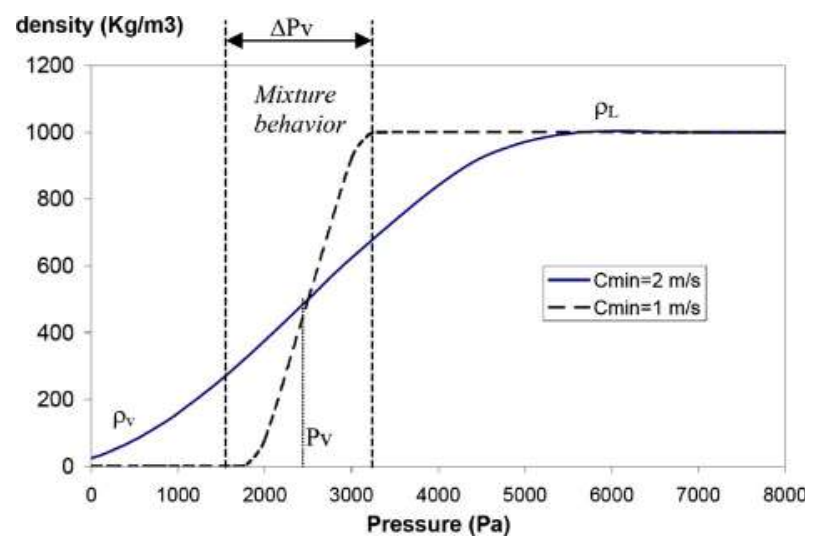

Fig. 1 Graph of the barotropic state law $\rho=\rho(P)$. Illustration of the speed of sound influence for water at $20^{\circ} \mathrm{C}$. For more information, see Ref. [8].

density. The equivalent void ratio $\alpha$ of the liquid-vapor mixture relates to the varying specific mass by $\rho=\alpha \rho_{v}+(1-\alpha) \rho_{l}$. In the mixture, the velocities of liquid and vapor phases are the same and we obtain only one set of equations for the mixture mass, momentum, or turbulence $(k-\varepsilon)$, written in their conservative form.

The void ratio $\alpha$ of the mixture depends on the local static pressure. As initially proposed by Delannoy and Kueny [6], we use a barotropic state law $\rho(P)$ to manage the relation between pressure and mixture density (Fig. 1). A smooth arbitrary law was chosen, $\rho$ rapidly varying between liquid density $\rho_{l}$ and vapor density $\rho_{v}$ when the local static pressure $P$ is around the vapor pressure $P_{v}$. The law is characterized by its maximum slope at $P=P_{v}$, which is related to the minimum speed of sound $c_{\min }$ in the two-phase homogeneous medium [8].

In the present work, based on experimental previous work concerning Venturi type sections (Stutz and Reboud $[15,16]$ ), the parameter $c_{\min }$ is taken equal to $2.35 \mathrm{~m} / \mathrm{s}$. The density ratio $\rho_{v} / \rho_{l}$ is imposed to be 0.1 . The compressibility of pure liquid and vapor phases is not taken into account. These choices were made to improve the robustness of the numerical code. Extensive numerical tests were carried out in other works to evaluate the influence of the speed of sound $c_{\min }$ and of the ratio $\rho_{v} / \rho_{l}$. Most important results obtained from these influence analyses are described in Refs. [17-19].

\section{Numerical Model}

The barotropic cavitation model was implemented in the commercial code FINE/TURBO ${ }^{\mathrm{TM}}$ developed by Numeca International. FINE/TURBO ${ }^{\mathrm{TM}}$ is a three-dimensional structured mesh code that solves the time dependent RANS equations, with artificial compressibility method. A detailed description of the initial code is given in Hakimi [20].

Time-accurate resolutions use the dual time stepping approach. Pseudotime derivative terms are added to the equations. They enable equation convergence at each physical time step. The initial set of mass and momentum equation can then be written under the following form:

$$
\iiint_{V} \frac{\partial \mathbf{U}}{\partial \tau} d V+\frac{\partial}{\partial t} \iiint_{V} \mathbf{U} d V+\iint_{S} \mathbf{F} \cdot \mathbf{d} \mathbf{S}=\iiint_{V} S T d V
$$

where

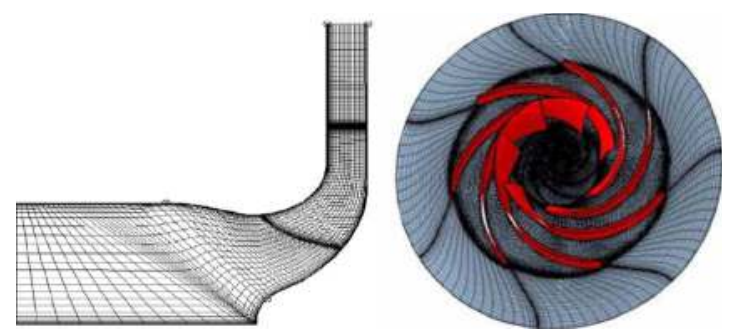

Fig. 2 Mesh of the SHF centrifugal pump

$$
\mathbf{U}=\left(\begin{array}{c}
\rho \\
\rho u \\
\rho v \\
\rho w
\end{array}\right)
$$

is the vector of the conservative variables, $\mathbf{F}=\mathbf{F}_{c}-\mathbf{F}_{v}$ notes the convective and diffusive fluxes across the surface $\mathbf{d} S=\mathbf{n} d S$, ST is the source term, and $\tau$ the pseudotime.

The space discretization is based on a finite volume approach. A second order central scheme is associated with two artificial dissipation terms, respectively, of second and fourth orders. The first one is activated in the strong pressure and density gradient areas. The other one is used in the whole domain, and it results in a second order space accuracy.

In the case of low-compressible or incompressible flows, the pseudotime derivatives are multiplied by a preconditioning matrix, based on the studies of Turkel [21], Choi and Merkle [22]. The modifications of the preconditioning technique developed for cavitating flows are detailed in Refs. [2,23].

The pseudotime integration is made by a four-step RungeKutta procedure. In noncavitating conditions, the code resorts to a multigrid strategy to accelerate the convergence. The different computations presented hereafter have been performed in the steady case, without taking into account the real time derivative term.

A more detailed description of the code is given in Refs. [1-3] A meaningful numerical work has been performed by Pouffary [23] to improve the preconditioner and the stability of numerical code for calculations of cavitating flows. It is worth noting that these numerical modifications led to major improvements in accuracy and numerical stability in comparison with our previous computations of cavitating flows in turbomachinery [7,9,24,25]. An illustration and quantification of these numerical improvements will be presented in Sec. 5 .

\section{Studied Geometries}

Centrifugal Pump. The first considered geometry is the SHF centrifugal pump tested by Combes and Archer [26]. The SHF pump has a shrouded seven-bladed centrifugal impeller. The scale and the calculation configuration are chosen as close as possible to the experimental conditions to simplify comparisons in cavitating conditions. The outlet radius is $330 \mathrm{~mm}$, the inlet pipe length is $200 \mathrm{~mm}$, the rotation speed equals to $3000 \mathrm{rpm}$, and the nominal flow rate is $0.157 \mathrm{~m}^{3} / \mathrm{s}$. Grid resolution tests have been done in cavitation-free conditions. After comparing pump head and pressure distribution on the blade obtained with different multiblock structured meshes (I and $\mathrm{H}$ meshes with 170,000, 250,000 and 500,000 cells), an I mesh of 250,000 cells for one blade-to-blade channel was chosen (Fig. 2). Yang-Shih's $k-\varepsilon$ turbulent model with extended wall functions is used [27].

Turbopump Inducers. To achieve high rotational speed and low inlet pressure, rocket engine pumps are equipped with an axial stage. Two different four-bladed inducers have been tested. Inducer 1 was designed by Snecma (Fig. 3(a)). The influence of 

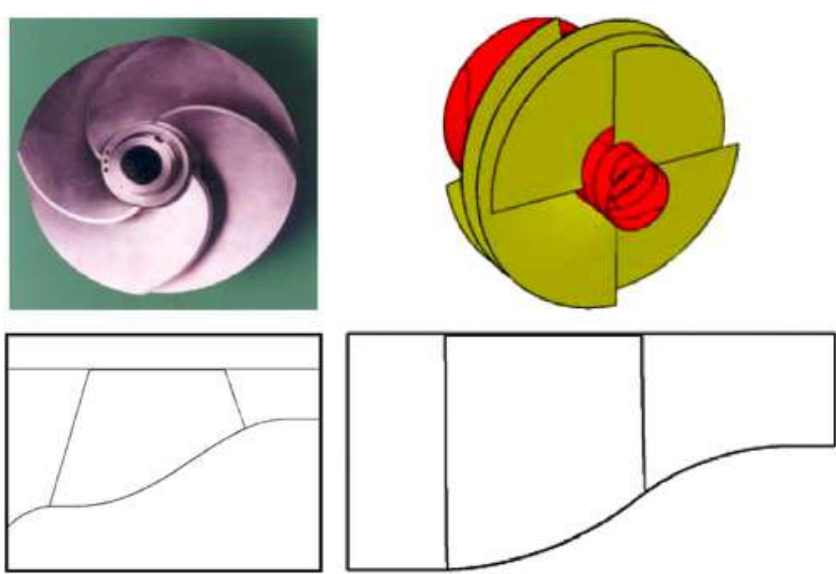

(a)

(b)

Fig. 3 Inducer geometries: (a) Inducer 1; (b) Inducer 2

the mesh and of the turbulence model (i.e., Baldwin-Lomax and three different $k-\varepsilon$ models) was firstly studied in noncavitating conditions, mainly by comparing the static pressure profile at the shroud with experimental measurements performed at different flow rates at the CREMHyG Laboratory (Grenoble, France). An example at nominal flow rate is given on Fig. 4. From these comparisons, a mesh of about 500,000 cells for one blade-to-blade channel was chosen, associated with a $k-\varepsilon$ model with wall functions [27]. Tip leakage was taken into account in noncavitating conditions, but to increase the numerical convergence speed, it was suppressed for the cavitating flow computations, by locally modifying the mesh. Analysis of the influence of the tip leakage on cavitating flows is in progress.

The geometry of Inducer 2 shows a pure radial leading edge shape (Fig. 3(b)) and has been experimentally studied at the University of Osaka [28]. The mesh has 250,000 cells for one bladeto-blade channel, and the tip leakage is not taken account of.

Simulation results presented in this paper for the three pump geometries are $3 \mathrm{D}$ and steady. Used meshes were chosen after a detailed study of mesh influence based on cavitation-free global performances of the inducer (head coefficient, torque, and efficiency).

\section{Analysis of Cavitating Flows}

Numerical Conditions. Steady calculations of cavitating and noncavitating flows were performed in these three geometries for different cavitation numbers.

In cavitation-free conditions, two sets of upstream and downstream boundary conditions have been compared in the case of Inducer 1 geometry: either the flow rate is imposed upstream and the static pressure downstream (with or without the radial equilibrium condition) or total pressure is imposed upstream and flow rate downstream. Influences of the rotation of hub and shroud boundaries upstream and downstream from the impeller have also been analyzed [23]. With a good convergence level of the computations, no major influences of the boundary conditions tested
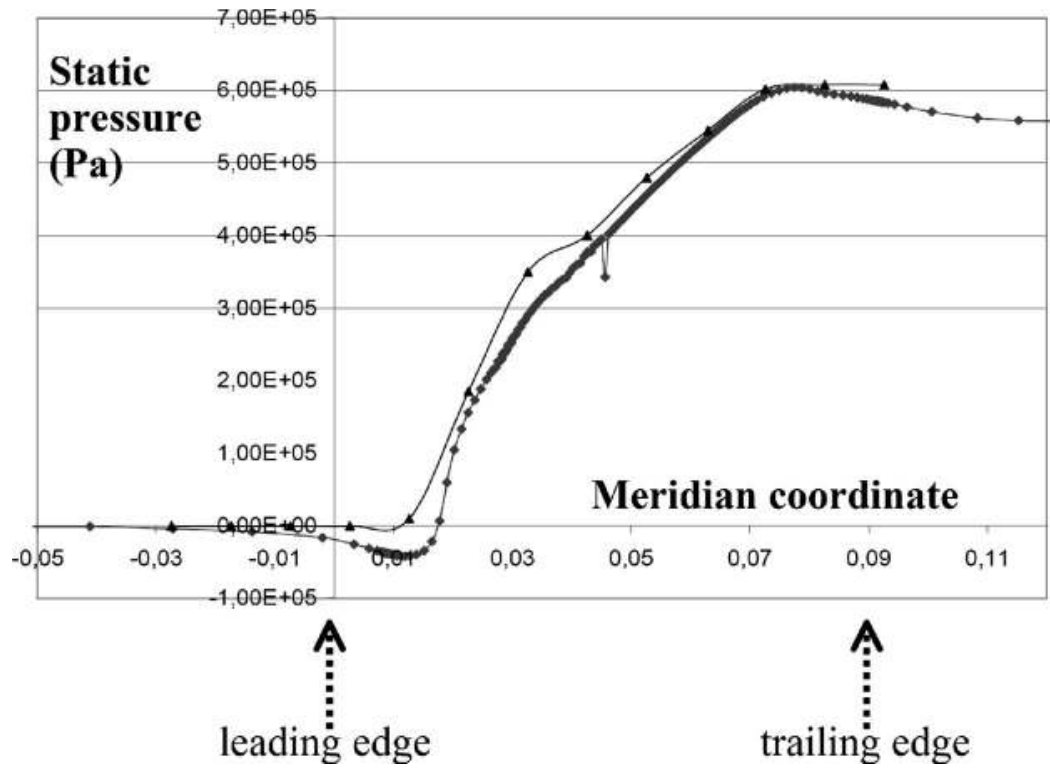

Fig. 4 Inducer 1 shroud static pressure at nominal flow rate (blue triangles, measurements; red rhombus, computation; $k-\varepsilon+$ wall functions). Noncavitating condition. Meridian coordinate is nondimensional. 


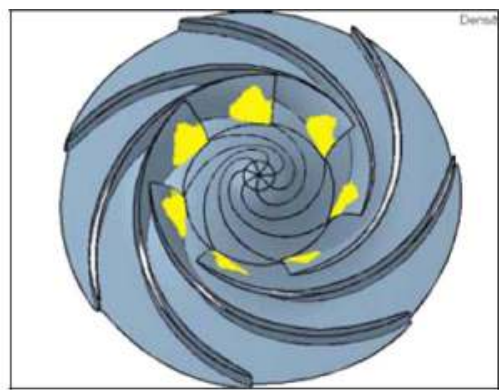

$\mathrm{NPSH}=9.5$

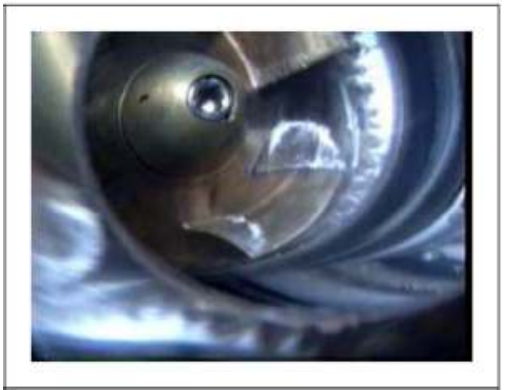

(a)
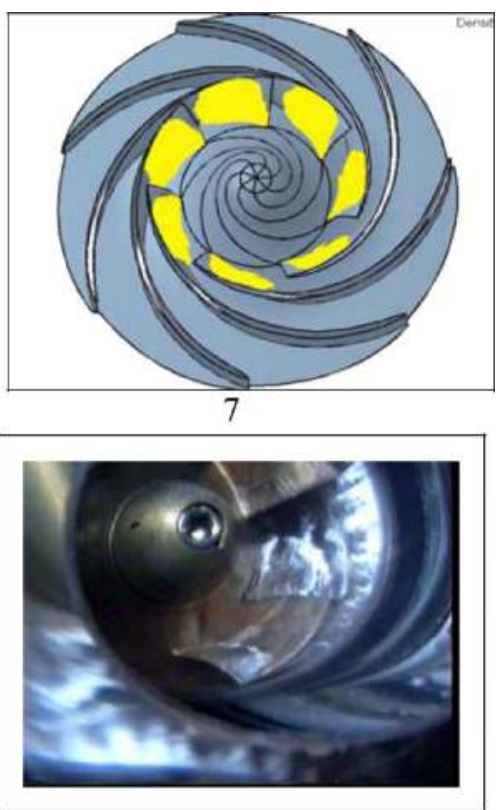

(b)

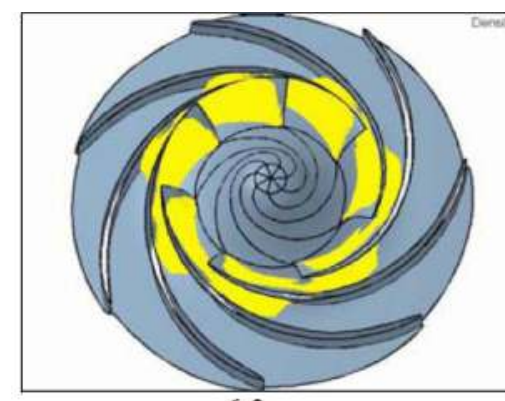

6.3

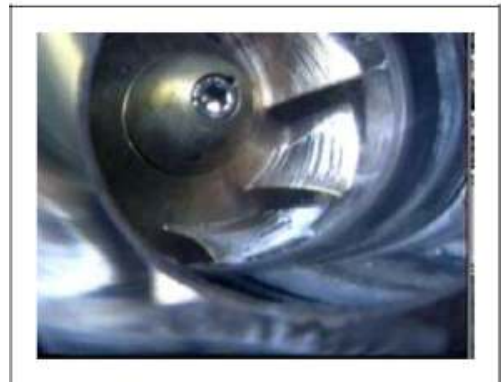

$(c)$

Fig. 5 SHF centrifugal pump, nominal flow rate, for decreasing NPSH $=\left(P_{\text {ref }}-P_{v}\right) /(\rho g) \approx 9.5 \mathrm{~m}, 7 \mathrm{~m}$, and $6.3 \mathrm{~m}$. The yellow color corresponds to a $5 \%$ equivalent void ratio. Comparison experiment/calculation of the suction side cavity extension. Experimental visualizations by Electricité de France [26].

were observed on the evaluation of global performances.

In cavitation conditions, the flow rate is imposed upstream and the static pressure downstream. The different analyzed cavitation numbers (or corresponding NPSH) are obtained by varying the pseudovapor pressure $P_{v}$ involved in the definition of cavitation number $\sigma$, while the downstream pressure is kept constant.

It is worth noting that each calculated steady state solution was carefully converged. Convergence criteria of cavitating flow calculations were based on the comparisons between inlet and outlet mass-flow rates (allowed maximum error $<0.4 \%$ ), on the analyses of the torque, the static and total heads obtained through numerical iterations (pseudotime integrations), and on the verification of the mass-flow rate through different sections inside the pumps, located in cavitating flow areas.

In relation to previous work [2], numerical modifications were performed by Pouffary [23], on the preconditioning technique and associated stability of the numerical algorithms, to deal with very large variations of compressibility of the liquid-vapor mixture in cavitating conditions. "Local velocity scaling" strategy was used for SHF pump calculations. For inducer computations, preconditioning coefficients were calculated as a function of the reference velocity $U_{\text {ref }}$ and of the barotropic state law. These improvements allowed a very good convergence level, even in the cases of very large cavitation structures and associated strong modifications of the pump head. Indeed, operating points in cavitating regime leading to a $10 \%$ head drop or more could be accurately simulated in the three pump geometries considered in this paper.

Centrifugal Pump. For the SHF centrifugal pump, the cavitating behavior obtained at nominal flow rate $Q n$ shows thin attached sheet cavities on the suction side of the impeller blades, their length increasing with the decreasing cavitation number (Fig. 5). Head begins to approximately drop when the cavitation sheet reaches the throat between two adjacent blades and rapidly decreases by more than $10 \%$ of its noncavitating value (Fig. 6). The simulated prediction of head breakdown is found for the critical NPSH close to $6.3 \mathrm{~m}$, which corresponds to the $\mathrm{NPSH}_{3 \%}$ experimentally found at Electricité de France [26]. Flow rates superior to the nominal flow rate lead to larger critical NPSH, head breakdown being observed at $1.22 Q n$ and $1.3 Q n$ for NPSH values, respectively, of about $18 \mathrm{~m}$ and $26 \mathrm{~m}$.

Simulation results obtained at nominal flow rate were analyzed in detail. It can be seen on Fig. 7 that the head drop can be mainly associated with a decrease of the torque (at the NPSH with $7 \%$ total head decrease, the calculated torque decreases by about $5 \%$ and the efficiency by $2 \%$ ).

The torque decrease can be illustrated by Fig. 8, where the static pressure distributions around a blade section at midspan are drawn for different NPSH values. When the net pressure $P_{\text {ref }}-P_{v}$ becomes smaller, a long sheet cavity develops along the blade suction side, reducing the suction effect near the blade leading edge, without significantly modifying the pressure distribution along the wetted surfaces, and thus clearly reducing the blade load. At head breakdown, it can be observed that cavitation also appears on the upstream part of the blade pressure side (NPSH $\sim 6.3$ ). Such observations can also be made along other sections of the pump blades.

To study the energy transfers within the pump, an analysis of

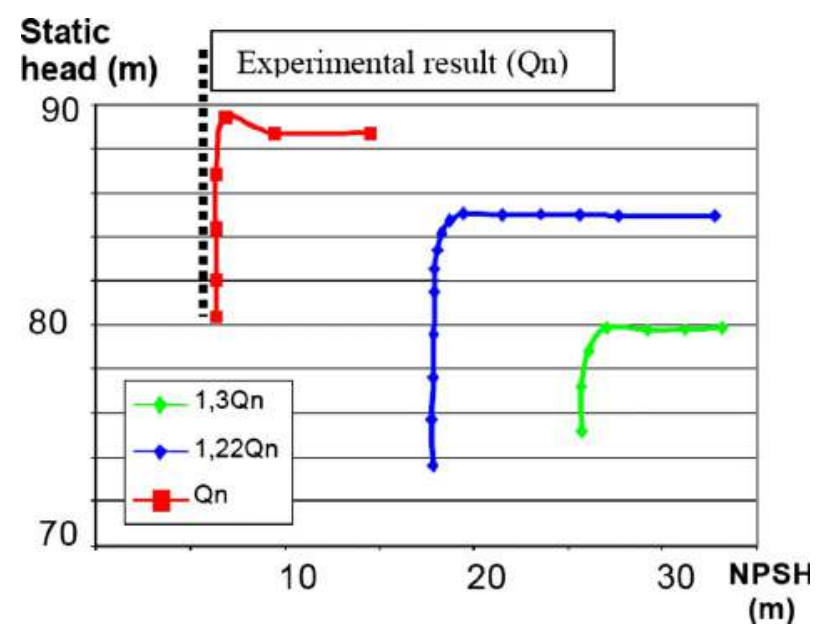

Fig. 6 Cavitation head drop curves at different flow rates 


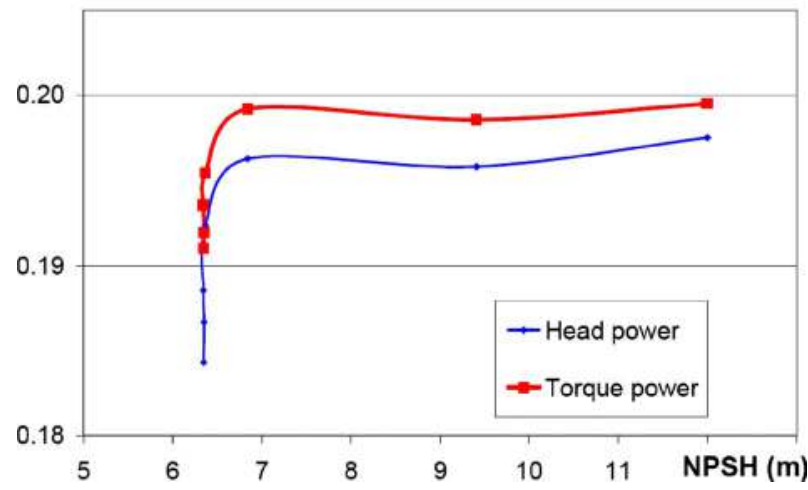

Fig. 7 Nominal flow rate. Evolution of mechanical and hydraulic power calculated; mechanical power $=T \omega$ hydraulic power $=\Delta P_{\text {tot }} \cdot Q$.

the flow has been performed in eight different flow sections, from Cut I near the blade leading edge to Cut VIII near the trailing edge (Fig. 9). First of all, the mass-flow averaged total pressure rise between successive sections is drawn. One can clearly see on Fig. 10 that the total pressure breakdown principally occurs in the upstream Sections I-III, while the downstream ones (IV-VIII) approximately remain unaffected by the development of cavitation. During the beginning of the head drop (Calculations 1 and 2), the total pressure rise between Cuts I and III increases, leading to the rise of global performance observed in Fig. 6 for these NPSH values. This phenomenon seems to be related to an inci-

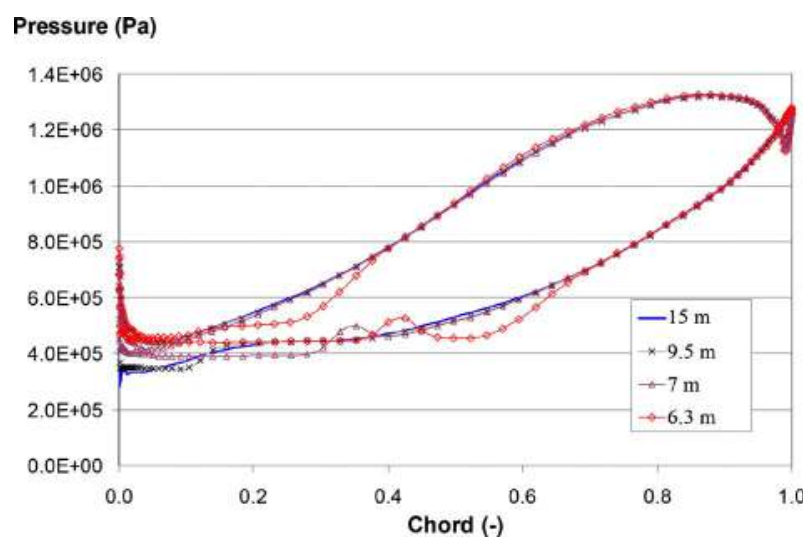

Fig. 8 Nominal flow rate. Blade load at midspan, for decreasing NPSH $\approx 15 \mathrm{~m}, 9.5 \mathrm{~m}, 7 \mathrm{~m}$, and $6.3 \mathrm{~m}$ (with about $7 \%$ total head drop).

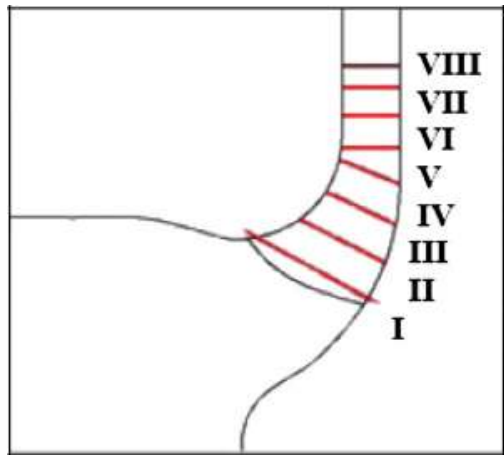

Fig. 9 Location of the analyzed flow sections in the SHF pump
Total pressure rise $(\mathrm{Pa})$

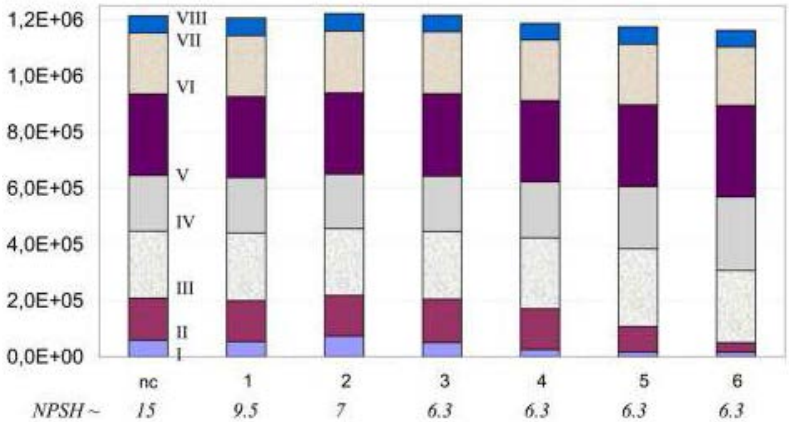

Fig. 10 Repartition of the total pressure rise between Sections 1 and VIII of the SHF pump, in noncavitating conditions (nc: left) and with decreasing downstream cavitation numbers (16). Total pressures are evaluated by mass-flow averaging in the cut plane. As the NPSH is calculated a posteriori from the upstream pressure resulting from the cavitating flow simulation, it takes into account the decreasing pump head associated with cavitation breakdown. The last four NPSH values, during final head drop, are found nearly constant, whereas the net downstream pressure continues to be decreased.

dence effect: The meridional flow velocity decreases due to the blockage effect of cavitation and this leads to a local rise of the effective angle of attack.

If one observes the meridian repartition of the total pressure rise corresponding to Calculations 3-6, one can note also, downstream of the cavitation sheet, an augmentation of the total pressure rise. This phenomenon of compensation is mainly observed between Cuts IV and VI.

For a better understanding, a local analysis of the influence of cavitation on the flow structure in the different sections has been also performed. In the present paper, the analysis of the secondary flows is based on the dimensionless relative helicity, drawn for the four upstream sections (I-IV): Fig. 11(a) illustrates the results concerning cavitation-free condition and Fig. 11(b) corresponds to a regime close to the cavitation breakdown. The blue colors represent the anticlockwise vorticity and the yellow to red ones indicate the clockwise vorticity. In noncavitating conditions, one can observe that the secondary flows in the rotating frame mainly develop along the blade suction side (blue color), progressively evolving to a hub vortex (Section IV). Clockwise vorticity (red color) can also be observed along the shroud.

In cavitating conditions (Fig. 11(b)), secondary flows are strongly modified along the cavitation sheet surface: in Section I, at the suction side, one observes a well organized structure of clockwise vorticity corresponding to the region of the cavitation sheet. In Section II, this structure disappears. The cavity wake generates a large anticlockwise structure, which seems to be related to the centrifugation of the cavity wake. In Sections III and $\mathrm{IV}$, these vorticity structures due to the presence of the cavity sheet progressively dissipate.

Inducer 1. An analogous analysis has been performed for the Inducer I geometry. Development of cavitation structures starts from the blade tip and rapidly extends along the leading edge to the hub side. Then the cavitation sheet length progressively increases until it reaches the throat (Fig. 12). The head drop abruptly occurs when the cavity enters into the blade-to-blade channel.

Comparison of the simulated cavitation performance chart with experiments performed at the CREMHyG Laboratory is drawn on Fig. 13. For a high cavitation number, the obtained simulated value is found about 5\% higher than the experimental inducer head, which can be mainly attributed to the fact that the tip leakage flow is not taken into account by numerical calculations. Successive cavitating flows were computed and a good convergence 

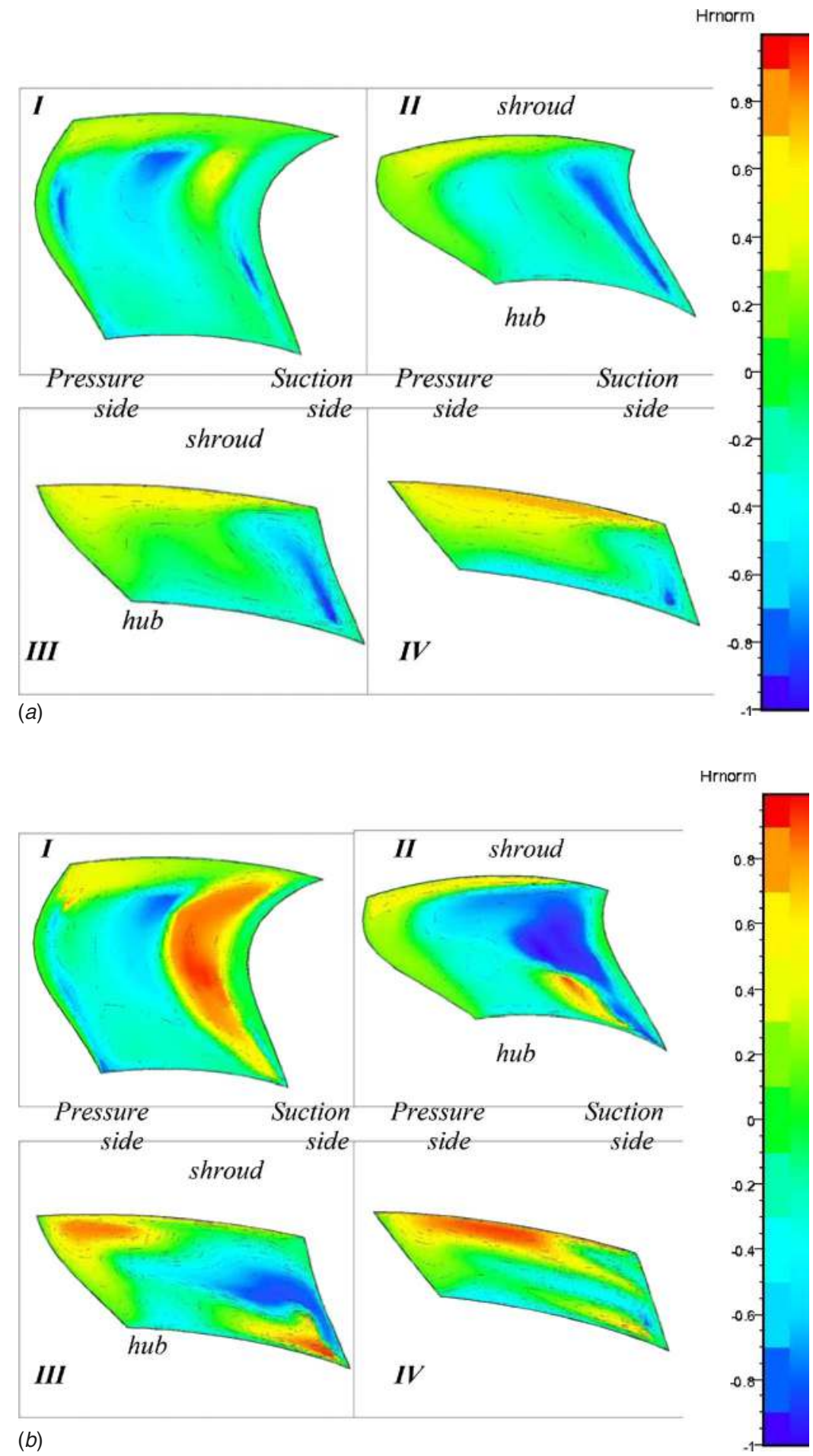

Fig. 11 (a) Secondary flows: Dimensionless relative helicity, varying from -1 (blue, maximum anticlockwise vorticity) to 1 (red, maximum clockwise vorticity). Noncavitating flow. Planes I to IV. (b) Secondary flows (dimensionless relative helicity). Cavitating flow corresponding to $7 \%$ total head decrease. Planes I to IV.

could be obtained even with more than $10 \%$ head loss. The critical cavitation number is very well predicted (Fig. 13) and the improvement of this prediction is significant with respect to the preceding code version [29].

According to the analyses done, detailed in Ref. [23], the inducer head drop seems to be mainly associated with a drop of the torque: For the critical cavitation number, the calculated torque decreases by about $10 \%$ of the noncavitating value, while hydraulic efficiency decreases by about $3 \%$.

Static pressure distributions around the blade are drawn on Fig. 14 in noncavitating and strongly cavitating conditions: The blade load decrease is mainly due to the modification of the pressure 

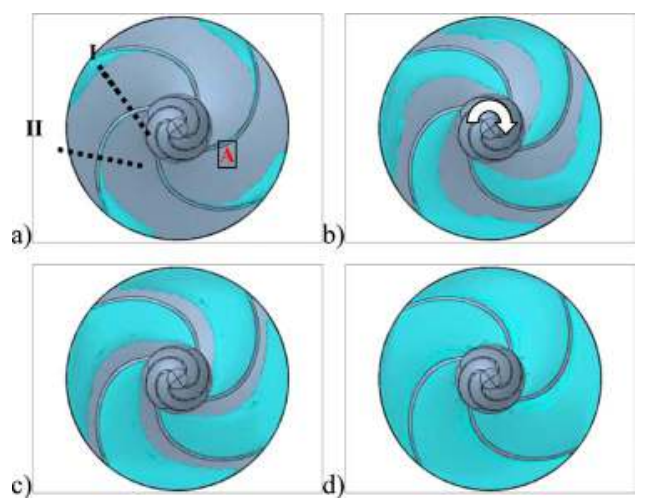

Fig. 12 Inducer 1, nominal flow rate, for decreasing cavitation number. The blue color corresponds to a $5 \%$ equivalent void ratio. Upstream cavitation numbers: (a) 0.0196; (b) 0.016 ; (c) 0.014 ; (d) 0.012 .

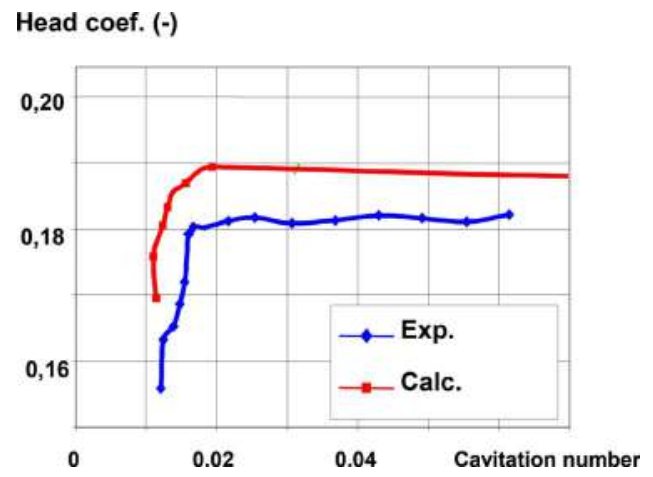

Fig. 13 Cavitation head drop curves at nominal flow rates. Comparisons between computations and experiments performed at the CREMHyG Laboratory (upstream cavitation number).

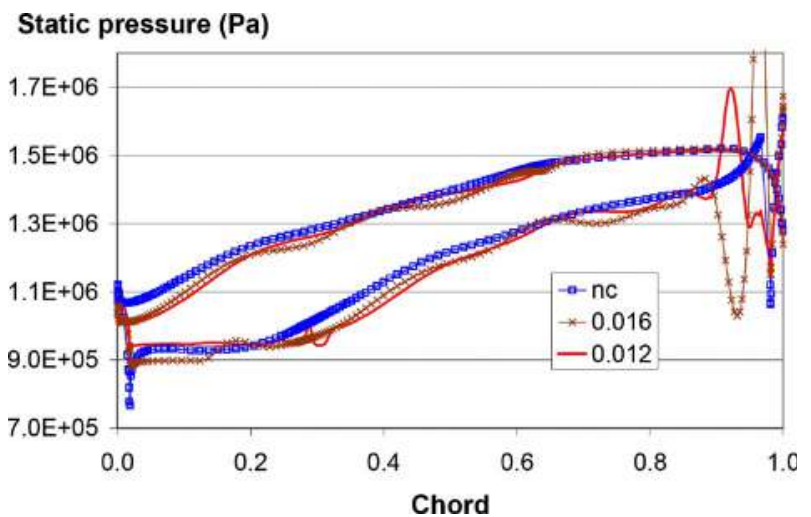

Fig. 14 Nominal flow rate. Inducer 1. Blade load at midspan, for decreasing cavitation numbers.

side distribution near the leading edge. The load reduction is observed when the cavitation sheet developed on the upstream blade suction side reaches the entry of the blade-to-blade channel. Perturbations of the trailing edge pressure distribution can also be observed. These perturbations can be associated with noticeable modifications of secondary flows, as will be discussed hereafter.

The mass-flow averaged total pressure rise between successive sections in Inducer 1 is drawn on Fig. 15. Analyzed sections are perpendicular to axial direction, as schematized in Fig. 21 for the Inducer 2 geometry. One can observe that the head decrease is mainly caused by the decrease in the upstream region.

The secondary flows in cut Sections I and II (presented in Fig. 12) are analyzed in Fig. 16. In noncavitating condition, an intense anticlockwise vorticity structure near the shroud is observed, which seems to be mainly due to the kinetic energy of the tip leakage flow. Another anticlockwise vorticity structure is observed near the suction side and a clockwise one is present close to the pressure side; both structures are related to the centrifugation of the boundary layer along the blades. There is no relevant vorticity effect in the middle of the analyzed sections.

In cavitating conditions (Fig. 16(b)), near the head breakdown, the secondary flow in Section I is slightly modified. On the other

\section{Total pressure rise $(\mathrm{Pa})$}

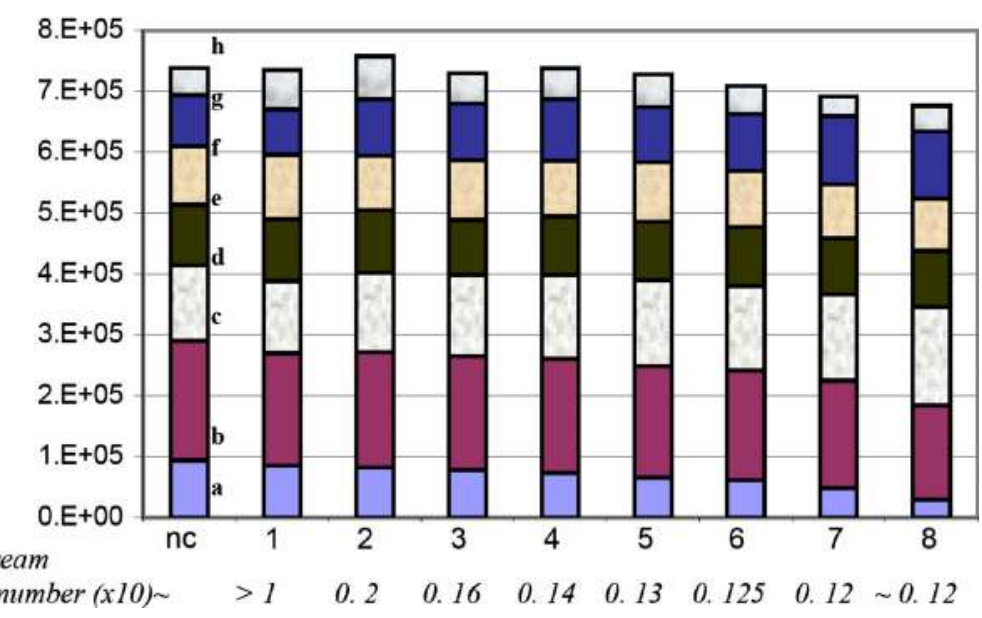

Fig. 15 Repartition of the total pressure rise between Sections " $a$ " and " $h$ " of Inducer 1, in noncavitating conditions (nc: left) and with decreasing downstream cavitation numbers (1-8). Total pressures are evaluated by mass-flow averaging in the cut plane. Sections "a"-" $h$ " are perpendicular to the axial direction, as schematized in Fig. 21 for the Inducer 2 geometry. As in Fig. 10, the last two upstream cavitation number values are found nearly constant for decreasing values of the downstream cavitation number. 


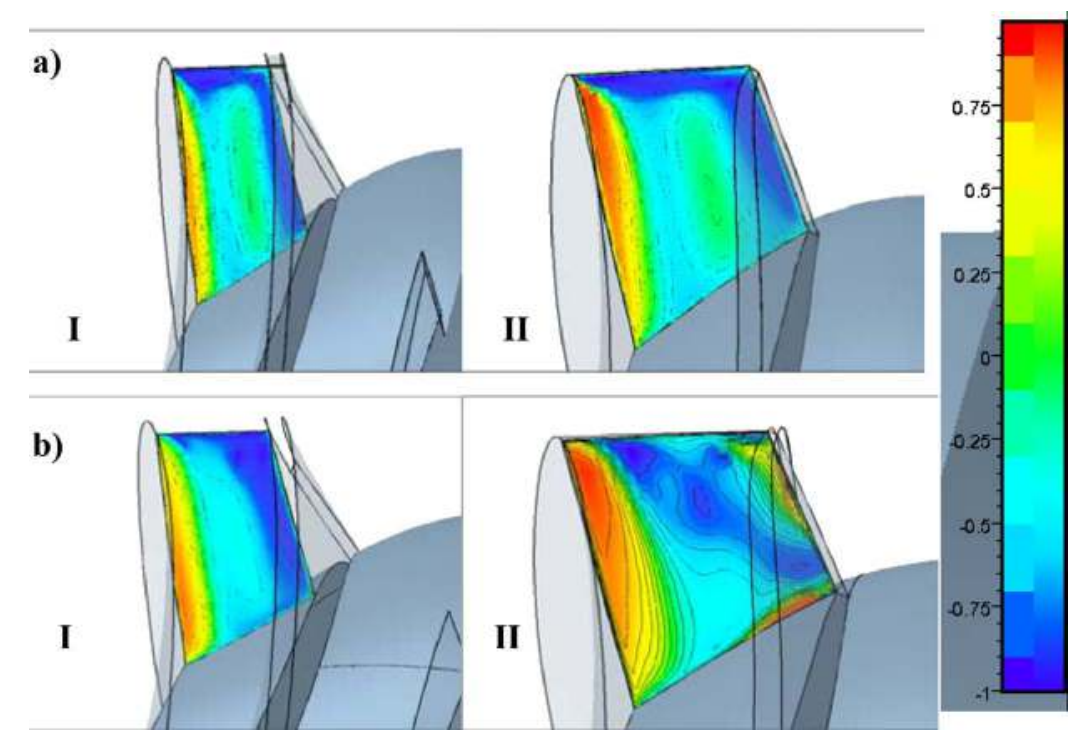

Fig. 16 Secondary flows (dimensionless relative helicity), and noncavitating (a) and cavitating (b) flows. Planes I and II.

hand, the outlet flow is highly influenced by the important cavity wake structure. This can be seen in Section II: the clockwise vorticity structure observed near the pressure side is amplified; the vorticity structure close to the suction side is convected through the middle of the section; a small secondary structure appears near the hub. Figure 16 shows that the downstream flow is highly influenced by the cavitation development: Increasing vorticity observed near the pressure side (left part of Plane II in Fig. 16(b)) interacts with the suction side in the trailing edge region of the inducer (right part of Plane II), and seems to lead to pressure distribution perturbations observed in Fig. 14 in this zone.

It is worth reminding that calculations performed under cavitating conditions do not take into account tip leakage. It is the reason why intense anticlockwise vorticity structure observed near the shroud in noncavitation condition (Fig. 16(a)) does not appear on Fig. 16(b). Improvements of methodology for tip leakage meshing, in association with multigrid strategy to accelerate the convergence of cavitating flow calculations, are needed to progress on secondary flow analyses and on the evaluation of the tip clearance effect for cavitating cases. Such improvements are still in progress.

Inducer 2. The second analyzed inducer geometry shows a quite different behavior in cavitating conditions. According to our numerical study, as illustrated by Fig. 17, the development of the

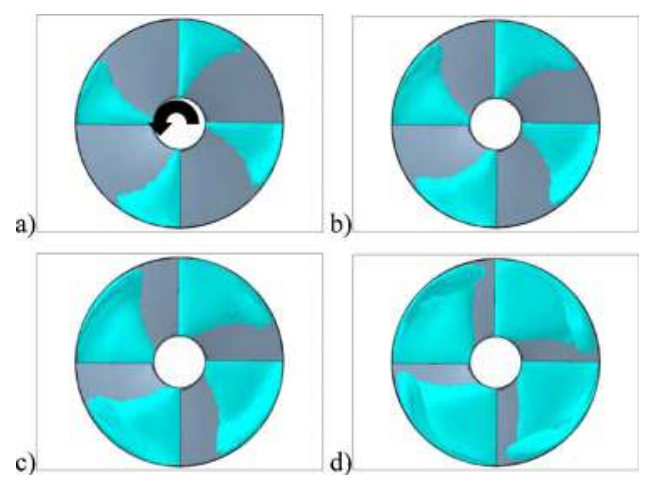

Fig. 17 Inducer 2, nominal flow rate, for decreasing upstream cavitation numbers: (a) 0.12 ; (b) 0.08 ; (c) 0.05 ; (d) 0.03 . The blue color corresponds to a $5 \%$ equivalent void ratio. cavitation structures appears much more pronounced at the inducer periphery. We can observe also a large backflow structure in front of the blades. On the blade suction side, the cavitation sheets show a triangular shape, increasing from hub to periphery, and merging with the cavitating backflow structure.

On the performance chart, illustrated by Fig. 18, the head drop much more gradually appears than for Inducer 1 . The last point calculated shows a $20 \%$ head drop, mainly due to the drop of the torque. The efficiency decreases by about $3 \%$.

The fact that the cavitation head drop is less abrupt can be explained by analyzing the blade load evolution drawn on Fig. 19, and comparing it to Fig. 14. In noncavitating condition, the blade loads of the two inducers appear very different: Inducer 2 blades are mainly loaded near the leading edge, while the load remains approximately constant along the Inducer 1 blades. In cavitating regime, the development of the sheet cavity from the leading edge of the Inducer 2 blade suction side gradually modifies the blade load, while in Inducer 1, the blade load decreases only when the cavity closure reaches the blade-to-blade passage.

It can be clearly seen on Fig. 20 that the main part of the total pressure rise in Inducer 2 is provided by the upstream part of the inducer. As a consequence, cavitation head drop mainly occurs due to a progressive decrease of the pressure rise between Sections I and II. The blade load at the downstream part of the inducer is not very significant and a compensation phenomenon cannot appear with this inducer design.

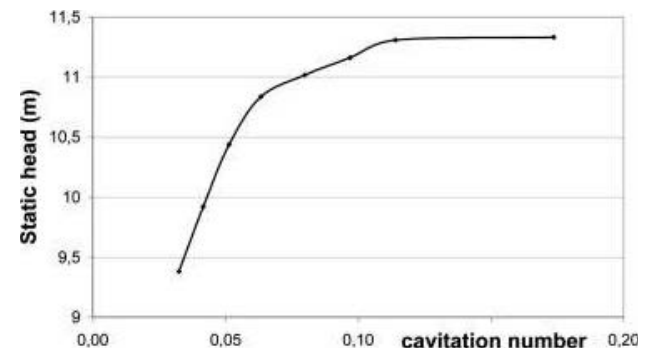

Fig. 18 Cavitation head drop curve. Nominal flow rate, Inducer 2. 


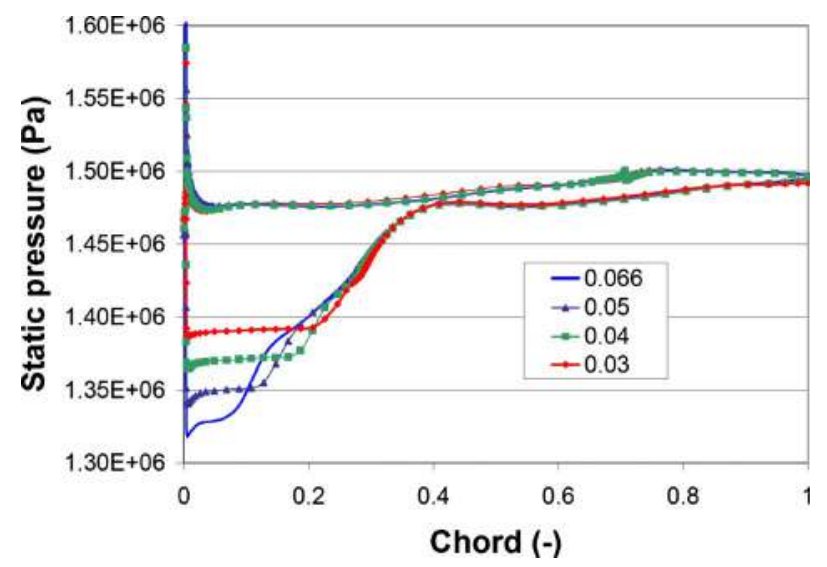

Fig. 19 Nominal flow rate. Inducer 2. Blade load at mid span, for decreasing cavitation numbers.

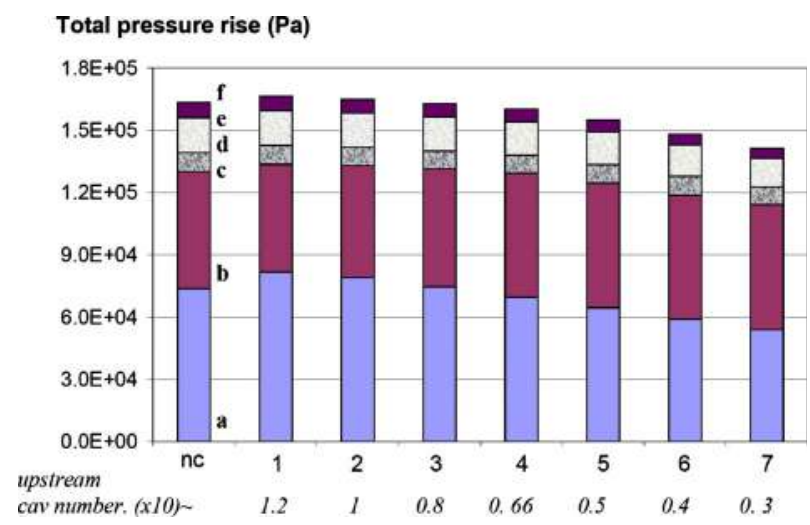

Fig. 20 Repartition of the total pressure rise between Sections "a"-"f" of Inducer 2, in noncavitating condition (nc: left) and with decreasing downstream cavitation numbers (1-7). Total pressures are evaluated by mass-flow averaging in the cut plane. Sections "a"-"f" locations are presented in Fig. 21.

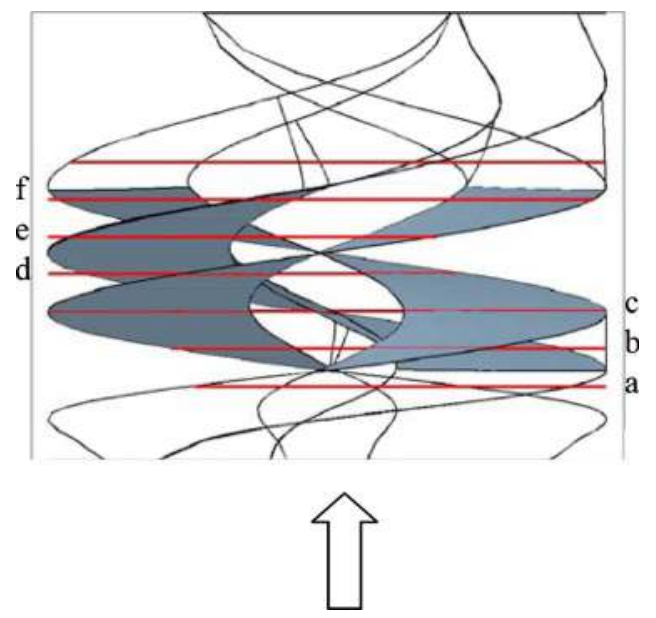

Fig. 21 Illustration of Planes "a"-"f" in the meridian view of Inducer 2

\section{Conclusion}

A numerical model of 3D cavitating flows has been developed to predict the cavitation behavior in turbomachinery. Compared to the previous studies $[7,9,24,25]$, thanks to recent improvements of numerical stability and algorithm reliability, very good conver- gence and accuracy of the simulation of cavitating flows in turbomachinery can now be obtained, even for operating conditions very close to the machine head drop (Fig. 13).

Simulation results were presented and analyzed for a centrifugal pump and two different four-bladed inducers. Noncavitating and cavitating conditions were investigated and compared. The vapor structure extensions and associated pump head breakdowns seem to be correctly simulated by the numerical model and favorably compare with available experimental results.

Local analyses of the results provide interesting information on the breakdown mechanisms.

- In the three geometries, head breakdown is clearly associated with a decrease of the torque provided by the impeller, through a strong variation of the blade load. Otherwise, only a small decrease of the machine efficiency can be associated with the development of the cavitation structures.

- An important difference between the cavitation behaviors of the two inducers can be observed, which can be explained by an analysis of their respective blade load and total pressure rise repartition.

- Local visualizations of the flow fields and their evolution with cavitation can also be obtained. For example, the analysis of dimensionless relative helicity maps gives useful information about secondary flows in the machines and enables the identification of the location and influence of the cavity wakes in the blade-to-blade channels.

The methodology of analysis proposed in the present paper shows that numerical simulation can provide useful information for the design of turbomachinery, and more particularly of inducers. Applications performed on unsteady flows are in progress and the first results presented in Refs. $[8,17]$ are very promising for the analysis and prediction of unstable cavitation behavior in turbomachinery.

Improvements of the cavitation model are also in development, mainly to take into account the thermodynamic behavior of fluids, such as hydrocarbons or cryogenic propellants.

\section{Acknowledgment}

This research was supported by the French space agency Centre National d'Etudes Spatiales (CNES) and Snecma. The authors wish also to express their gratitude to Electricité de France and CREMHyG for providing experimental results, to Professor Tsujimoto for providing the Inducer 2 geometry, and to Numeca International for its cooperation to the development of the numerical code.

\section{Nomenclature}

$$
\begin{aligned}
& c_{\min }=\text { minimum speed of sound in } \\
& H r_{\text {norm }}=\frac{\mathbf{W} \cdot \operatorname{rot}(\mathbf{W})}{\|\mathbf{W}\| \cdot\|\operatorname{rot}(\mathbf{W})\|}=\text { dimensionless relative helicity } \\
& L_{\text {ref }}=\text { geometry reference length }(\mathrm{m}) \\
& P_{\text {ref }}=\text { reference pressure }=\text { total up- } \\
& \text { stream pressure }(\mathrm{Pa}) \\
& P_{\text {up }}\left(P_{\mathrm{dw}}\right)=\text { static upstream (downstream) } \\
& \text { pressure }(\mathrm{Pa}) \\
& P_{v}=\text { vapor pressure }(\mathrm{Pa}) \\
& Q=\text { flow rate }\left(\mathrm{m}^{3} / \mathrm{s}\right) \\
& t=\text { physical time (s) } \\
& T=\text { torque }(\mathrm{N} \mathrm{m}) \\
& u, v, w=\text { velocity components }(\mathrm{m} / \mathrm{s}) \\
& U_{\text {ref }}=\text { reference velocity (generally } \\
& \left.U_{\text {blade_tip }}\right)(\mathrm{m} / \mathrm{s}) \\
& \mathbf{W}=\text { relative velocity vector (rotat- } \\
& \text { ing frame })(\mathrm{m} / \mathrm{s}) \\
& \alpha=\text { void ratio }
\end{aligned}
$$




$$
\begin{aligned}
\Delta P_{\text {tot }}= & \text { total pressure rise in the pump } \\
& (\text { Pa }) \\
\rho=\alpha \rho_{v}+(1-\alpha) \rho_{l}= & \text { mixture density }\left(\mathrm{kg} / \mathrm{m}^{3}\right) \\
\rho_{l}\left(=\rho_{\text {ref }}\right), \rho_{v}= & \text { liquid }(=\text { ref }), \text { vapor density } \\
& \left(\mathrm{kg} / \mathrm{m}^{3}\right) \\
\sigma_{\text {up }}=\left(P_{\text {up }}-P_{v}\right) /\left(\rho V_{\mathrm{ref}}^{2} / 2\right)= & \text { upstream cavitation number } \\
\sigma_{\mathrm{dw}}=\left(P_{\mathrm{dw}}-P_{v}\right) /\left(\rho V_{\mathrm{ref}}^{2} / 2\right)= & \text { downstream cavitation number } \\
\omega= & \text { pump rotation speed }(\mathrm{rad} / \mathrm{s}) \\
\mathrm{NPSH}= & \text { Net pressure suction head } \\
& \left(P_{\text {ref }} P_{v}\right) /(\rho g)(\mathrm{m}) \\
\tau= & \text { pseudotime }(\mathrm{s})
\end{aligned}
$$

\section{References}

[1] Coutier-Delgosha, O., Fortes-Patella, R., Reboud, J. L., Hakimi, N., and Hirsch, C., 2005, "Numerical Simulation of Cavitating Flow in 2D and 3D Inducer Geometries," Int. J. Numer. Methods Fluids, 48(2), pp. 135-167.

[2] Coutier-Delgosha, O., Fortes-Patella, R., Reboud, J. L., Hakimi, N., and Hirsch, C., 2005, "Stability of Preconditioned Navier-Stokes Equations Associated With a Cavitation Model," Comput. Fluids, 34(3), pp. 319-349.

[3] Pouffary, B., Fortes-Patella, R., and Reboud, J. L., 2003, "Numerical Simulation of Cavitating Flow Around a 2D Hydrofoil: A Barotropic Approach," Fifth International Symposium on Cavitation, Osaka, Japan.

[4] Kunz, R. F., Boger, D. A., Stinebring, D. R., Chyczewski, T. S., Lindau, J. W., and Gibeling, H. J., 2000, "A Preconditioned Navier-Stokes Method for TwoPhase Flows With Application to Cavitation," Comput. Fluids, 29(8), pp. 849875.

[5] Medvitz, R. B., Kunz, R. F., Boger, D. A., Lindau, J. W., Yocum, A. M., and Pauley, L. L., 2001, "Performance Analysis of Cavitating Flow in Centrifugal Pumps Using Multiphase CFD," ASME-FEDSM '01, New Orleans.

[6] Delannoy, Y., and Kueny, J. L., 1990, "Two Phase Flow Approach in Unsteady Cavitation Modelling," Cavitation and Multiphase Flow Forum, ASME-FED, Vol. 98, pp. 153-158.

[7] Coutier-Delgosha, O., Fortes-Patella, R., Reboud, J. L., Hofmann, M., and Stoffel, B., 2003, "Experimental and Numerical Studies in a Centrifugal Pump With 2D-Curved Blades in Cavitating Conditions," ASME J. Fluids Eng., 125(6), pp. 970-978.

[8] Pouffary, B., Fortes-Patella, R., Reboud, J. L., and Lambert, P. A., 2008, "Numerical Analysis of Cavitation Instabilities in Inducer Blade Cascade," ASME J. Fluids Eng., 130, p. 041302.

[9] Coutier-Delgosha, O., Morel, P., Fortes-Patella, R., and Reboud, J. L., 2005, "Numerical Simulation of Turbopump Inducer Cavitating Behavior," Int. J. Rotating Mach., 2, pp. 135-142.

[10] Ait-Bouziad, Y., Farhat, M., Guennoun, F., Kueny, J. L., Avellan, F., and Miyagawa, K., 2003, "Physical Modelling and Simulation of Leading Edge Cavitation, Application to an Industrial Inducer," Fifth International Symposium on Cavitation, Osaka, Japan.

[11] Ait-Bouziad, Y., Farhat, M., Kueny, J. L., Avellan, F., and Miyagawa, K. 2004, "Experimental and Numerical Cavitation Flow Analysis of an Industrial Inducer," 22th IARH Symposium on Hydraulic Machinery and Systems, Stockholm, Sweden
[12] Mejri, I., Bakir, F., Rey, R., and Belamri, T., 2006, "Comparison of Computational Results Obtained From a Homogeneus Cavitation Model With Experimental Investigations of Three Inducers," ASME J. Fluids Eng., 128, pp. $1308-1323$.

[13] Hosangadi, A., and Ahuja, V., 2001, "Simulations of Cavitating Flows Using Hybrid Unstructured Meshes," ASME J. Fluids Eng., 123, pp. 331-340.

[14] Hosangadi, A., Ahuja, V., and Ungewitter, R. J., 2006, "Numerical Study of a Flat Plate Inducer: Comparison of Performance in Liquid Hydrogen and Water," Sixth International Symposium on Cavitation, CAV2006, Wageningen, The Netherlands, September.

[15] Stutz, B., and Reboud, J. L., 1997, "Two-Phase Flow Structure of Sheet Cavitation," Phys. Fluids, 9(12), pp. 3678-3686.

[16] Stutz, B., and Reboud, J. L., 2000, "Measurements Within Unsteady Cavitation," Exp. Fluids, 29, pp. 545-552.

[17] Fortes-Patella, R., Coutier-Delgosha, O., Perrin, J., and Reboud, J. L., 2007, "A Numerical Model to Predict Unsteady Cavitating Flow Behaviour in Inducer Blade Cascades," ASME J. Fluids Eng., 129, pp. 128-135.

[18] Rolland, J., Boitel, G., Barre, S., and Fortes-Patella, R., 2006, "Experiments and Modelling of Cavitating Flows in Venturi, Part I.: Stable Cavitation," Sixth International Symposium on Cavitation, CAV2006, Wageningen, The Netherlands, September.

[19] Fortes-Patella, R., Barre, S., and Reboud, J.-L., 2006, "Experiments and Modelling of Cavitating Flows in Venturi, Part II: Unsteady Cavitation," Sixth International Symposium on Cavitation, CAV2006, Wageningen, The Netherlands, September.

[20] Hakimi, N., 1997, "Preconditioning Methods for Time Dependent NavierStokes Equations," Ph.D. thesis, Vrije University, Brussels.

[21] Turkel, E., 1987, "Preconditioning Methods for Solving the Incompressible and Low Speed Compressible Equations," J. Comput. Phys., 72, pp. 277-298.

[22] Choi, D., and Merkle, C. L., 1993, "The Application of Preconditioning in Viscous Flows," J. Comput. Phys., 105, pp. 207-223.

[23] Pouffary, B., 2004, "Simulation Numérique D'écoulements 2d/3d Cavitants, Stationnaires et Instationnaires: Analyse Spécifique Pour Les Turbomachines," $\mathrm{Ph} . \mathrm{D}$. thesis, Grenoble, France.

[24] Coutier-Delgosha, O., Pouffary, B., Fortes-Patella, R., Reboud, J. L., Archer, A., and Combes, J.-F., 2002, "Cavitation Performance of a Centrifugal Pump: Numerical and Experimental Investigations," 21st IAHR Symposium on Hydraulic Machinery and Systems, Lausanne, September.

[25] Pouffary, B., Coutier-Delgosha, O., Fortes-Patella, R., Reboud, J. L., Laffite, S., and Nguyen Duc, J.-M., 2002, "Evaluation of the Effects of Cavitation on the Flow in a Centrifugal Pump: Analysis of the Breakdown Mechanisms," 21 st IAHR Symposium on Hydraulic Machinery and Systems, Lausanne, September.

[26] Combes, J. F., and Archer, A., 2000, "Etude de la cavitation dans la pompe SHF," Coll. Machines Hydrauliques: Instationnarités et Effets Associés, Société Hydrotechnique de France, Chatou, France.

[27] Hakimi, N., Hirsch, C., and Pierret, S., 2000, "Presentation and Application of a New Extended $k-\varepsilon$ Model With Wall Functions," ECCOMAS, Barcelona, September.

[28] Yoshida, Y., Tsujimoto, Y., Kataoka, D., Horiguchi, H., and Wahl, F., 2001, "Effects of Alternate Leading Edge Cutback on Unsteady Cavitation in 4-Bladed Inducers," ASME J. Fluids Eng., 123, pp. 762-770.

[29] Coutier-Delgosha, O., 2001, "Modélisation des écoulements cavitants: Etude des comportements instationnaires et application aux turbomachines," Ph.D thesis, Grenoble, France. 